\title{
MATHEMATICAL INSTANTONS WITH MAXIMAL ORDER JUMPING LINES
}

\author{
A. Prabhakar RaO
}

\begin{abstract}
A mathematical instanton bundle on $\mathbf{P}^{3}$ (over an algebraically closed field) is a rank two vector bundle $\mathcal{E}$ on $\mathbf{P}^{3}$ with $c_{1}=0$ and with $H^{0}(\mathcal{E})=H^{1}(\mathcal{E}(-2))=0$. Let $c_{2}(\mathcal{E})=n$. Then $n>0$. A jumping line of $\mathcal{E}$ of order $a,(a>0)$, is a line $\ell$ in $\mathbf{P}^{3}$ on which $\mathcal{E}$ splits as $\mathcal{O}_{\ell}(-a) \oplus \mathcal{O}_{\ell}(a)$. It is easy to see that the jumping lines of $\mathcal{E}$ all have order $\leq n$. We will say that $\mathcal{E}$ has a maximal order jumping line if it has a jumping line of order $n$. Our goal is to show that such an $\mathcal{E}$ is unobstructed in the moduli space of stable rank two bundles, i.e., $H^{2}(\mathcal{E} \otimes \mathcal{E})=0$. The technique can be slightly extended. We show that when $c_{2}=5$, any $\mathcal{E}$ with a jumping line of order 4 is unobstructed. We describe at the end how mathematical instantons with maximal order jumping lines arise and estimate the dimension of this particular smooth locus of bundles.
\end{abstract}

It is known that every mathematical instanton bundle on $\mathbf{P}^{3}$ with $c_{2} \leq 4$ is unobstructed $([\mathbf{L}])$. In $[\mathbf{H}]$, it is shown that bundles built by the Serre construction from the union of $n+1$ skew lines and the bundles built from elliptic curves in $\mathbf{P}^{3}$ of degree $n+4$ are unobstructed (with $c_{2}=n$.) The $S U(2)$ instanton bundles obtained from physics are also known to be unobstructed using analytic arguments $([\mathbf{D}-\mathbf{V}])$. Recently Nüßler and Trautmann $[\mathbf{N}-\mathbf{T}]$ have shown that any mathematical instanton with a section in degree 1 is unobstructed, extending the case of bundles obtained from skew lines and also extending a result of Hirschowitz and Narasimhan on 't Hooft bundles [H-N]. A preprint of Ancona and Ottaviani ([A-O]) produces a singular point on the moduli space of stable bundles with $c_{1}=0, c_{2}=5$. This singular point is in the closure of the open set of mathematical instantons but is not itself a mathematical instanton bundle. Still open is the general

Question: for fixed Chern classes $c_{1}=0, c_{2}=n$, is the moduli space $\mathcal{M}_{m i}(0, n)$ of mathematical instanton bundles on $\mathbf{P}^{3}$ irreducible and smooth?

A construction of stable bundles on $\mathbf{P}^{3}$ (not necessarily mathematical instanton) with jumping lines of large order was first done by C. Peskine. 
In particular, he produced some examples of mathematical instantons with jumping lines of maximal order. The set of all mathematical instantons with jumping lines of maximal order turns out to be a locus of dimension $6 n+2$ (when $n \geq 3$ ) inside an $8 n-3$-dimensional component of $\mathcal{M}_{m i}(0, n)$. (In fact, $\mathcal{M}_{m i}(0, n)$ is smooth along this locus.) By comparison $([\mathbf{H}])$, the locus of mathematical instantons obtained from $n+1$ skew lines is of dimension $5 n+4$, and the locus obtained from elliptic curves of degree $n+4$ has dimension $4 n+16$ (for large $n$ ).

(Since writing an earlier version of this paper, it has been brought to my attention that M. Skiti has also worked on this locus of mathematical instantons. His results announced in $[\mathbf{S}]$ overlap with the calculations of the last section and he has communicated to me that he has proved unobstructedness for these bundles by a different method.)

\section{$\S 1$.}

Consider $\mathbf{P}^{3}$ over an algebraically closed field of characteristic not equal to 2 . A stable bundle $\mathcal{E}$ of rank two with $c_{1}=0$ on $\mathbf{P}^{3}$ is called a mathematical instanton (also a Yang-Mills bundle in the literature) if $H^{1}(\mathcal{E}(-2))=0$. Much of the literature treats these bundles only in characteristic zero. However results we will use (from $[\mathbf{L}]$ for example) are valid over characteristic not equal to 2 even though not explicitly declared as such.

A mathematical instanton bundle $\mathcal{E}$ with $c_{1}=0, c_{2}=n$ possesses a minimal monad:

$$
0 \rightarrow n \mathcal{O}_{\mathbf{P}^{3}}(-1) \stackrel{\alpha}{\rightarrow}(2 n+2) \mathcal{O}_{\mathbf{P}^{3}} \stackrel{\beta}{\rightarrow} n \mathcal{O}_{\mathbf{P}^{3}}(1) \rightarrow 0 .
$$

Then $\beta:(2 n+2) S \rightarrow n S(1)$ is a minimal presentation for $H_{*}^{1}(\mathcal{E})$ as an S-module where $S=k\left[X_{0}, X_{1}, X_{2}, X_{3}\right]$. In particular, $\beta$ has no column of zeroes. $\mathcal{G}=\operatorname{ker}(\beta)$ is a vector bundle of rank $n+2$ on $\mathbf{P}^{3}$. Since

$$
0 \rightarrow n \mathcal{O}_{\mathbf{P}^{3}}(-1) \stackrel{\alpha}{\rightarrow} \mathcal{G} \rightarrow \mathcal{E} \rightarrow 0,
$$

we get the Eagon-Northcott exact sequence

$$
0 \rightarrow \wedge^{2}\left(n \mathcal{O}_{\mathbf{P}^{3}}(-1)\right) \rightarrow n \mathcal{O}_{\mathbf{P}^{3}}(-1) \otimes \mathcal{G} \rightarrow S_{2} \mathcal{G} \rightarrow S_{2} \mathcal{E} \rightarrow 0
$$

Hence $H^{2}\left(S_{2} \mathcal{G}(-i)\right) \cong H^{2}\left(S_{2} \mathcal{E}(-i)\right), i=1,0,-1,-2, \ldots$

We also have (using $S_{2}\left(\mathcal{G}^{\vee}\right) \cong\left(S_{2} \mathcal{G}\right)^{\vee}$ in characteristic $\neq 2$ )

$0 \rightarrow S_{2} \mathcal{G} \rightarrow S_{2}\left((2 n+2) \mathcal{O}_{\mathbf{P}^{3}}\right) \rightarrow(2 n+2) \mathcal{O}_{\mathbf{P}^{3}} \otimes n \mathcal{O}_{\mathbf{P}^{3}}(1) \stackrel{B}{\longrightarrow} \wedge^{2}\left(n \mathcal{O}_{\mathbf{P}^{3}}(1)\right) \rightarrow 0$.

Hence $H_{*}^{2}\left(S_{2} \mathcal{G}\right)=\operatorname{cok} H_{*}^{0}(B)$. 
Now $0 \rightarrow \mathcal{O}_{\mathbf{P}^{3}} \rightarrow \mathcal{E} \otimes \mathcal{E} \rightarrow S_{2} \mathcal{E} \rightarrow 0$. Thus the cohomology modules describing obstructions to $\mathcal{E}$ in the moduli space are given as follows: Construct $B:(2 n+2) \mathcal{O}_{\mathbf{P}^{3}} \otimes n \mathcal{O}_{\mathbf{P}^{3}}(1) \rightarrow \wedge^{2}\left(n \mathcal{O}_{\mathbf{P}^{3}}(1)\right) \rightarrow 0$ out of the monad map $(2 n+2) \mathcal{O}_{\mathbf{P}^{3}} \stackrel{\beta}{\rightarrow} n \mathcal{O}_{\mathbf{P}^{3}}(1) \rightarrow 0$. Let $M$ be the module $\operatorname{cok}\left(H_{*}^{0}(B)\right) . M$ has $n(n-1) / 2$ generators in degree -2 .

$H^{2}(\mathcal{E} \otimes \mathcal{E}(-2))$ is a quotient of $M_{-2}$.

$H^{2}(\mathcal{E} \otimes \mathcal{E}(-1)) \cong M_{-1}$.

$H^{2}(\mathcal{E} \otimes \mathcal{E}) \cong M_{0}$, etc.

This discussion essentially follows Le Potier $[\mathbf{L}]$ where he gives a finer analysis of $H^{2}(\mathcal{E} \otimes \mathcal{E}(-2))$. Le Potier also shows that for any linear form $H$ on $\mathbf{P}^{3}$, the multiplication maps $H^{2}(\mathcal{E} \otimes \mathcal{E}(-2)) \stackrel{H}{\longrightarrow} H^{2}(\mathcal{E} \otimes \mathcal{E}(-1)) \stackrel{H}{\longrightarrow}$ $H^{2}(\mathcal{E} \otimes \mathcal{E}) \stackrel{H}{\longrightarrow} \ldots$ are surjective. Hence also the multiplication maps $M_{-2} \stackrel{H}{\longrightarrow}$ $M_{-1} \stackrel{H}{\longrightarrow} M_{0} \stackrel{H}{\longrightarrow} \ldots$ are surjective.

Suppose that $(2 n+2) \mathcal{O}_{\mathbf{P}^{3}}$ is split as $a \mathcal{O}_{\mathbf{P}^{3}} \oplus b \mathcal{O}_{\mathbf{P}^{3}}$, giving $\beta=\left(\phi_{1}, \phi_{2}\right)$. This induces a splitting of $B$ as $B=\left(F_{1}, F_{2}\right)$. We have the surjective maps of $S$ modules

$$
\wedge^{2}(n S(1)) \rightarrow N \rightarrow M
$$

where $S=k\left[X_{0}, X_{1}, X_{2}, X_{3}\right]$ and $N=\operatorname{cok}\left(H_{*}^{0}\left(F_{1}\right)\right)$.

We will be interested in the situation when $\phi_{1}$ and hence $F_{1}$ are matrices involving only two variables, say $X_{0}, X_{1}$. In this case, let $R$ be the subring of $S$ generated by these variables. The fact that all multiplication maps on $M$ by any form $H$ are surjective tells us that when $M$ is viewed as a graded $R$ module via the natural inclusion of $R$ in $S, M$ has no new generators; i.e., $M$ is a quotient of $\wedge^{2}(n R(1))$. Therefore the dimensions of the graded pieces of $M$ are bounded above by the dimensions of the graded pieces of $N_{R}$, where $N_{R}$ is the cokernel of $F_{1}$ viewed as a map from $a R \otimes n R(1) \rightarrow \wedge^{2}(n R(1))$.

To find good choices for $\phi_{1}$, hence $F_{1}$, we look at jumping lines of $\mathcal{E}$. On any line $\ell$, the restriction of $\mathcal{G}$ to $\ell, \mathcal{G}_{\ell}$, splits as $\oplus \mathcal{O}_{\ell}\left(-a_{i}\right)$ where each $a_{i} \geq 0$ and their sum is $n$. If $\mathcal{E}_{\ell}$ splits as $\mathcal{O}_{\ell}(-a) \oplus \mathcal{O}_{\ell}(a),(a \geq 0)$, we have

$$
0 \rightarrow n \mathcal{O}_{\ell}(-1) \stackrel{\alpha_{\ell}}{\longrightarrow} \oplus \mathcal{O}_{\ell}\left(-a_{i}\right) \rightarrow \mathcal{O}_{\ell}(-a) \oplus \mathcal{O}_{\ell}(a) \rightarrow 0
$$

Since the left hand map $\alpha_{\ell}$ is an inclusion of vector bundles on $\ell$, we see that at least $n$ of the $a_{i}$ 's are $\leq 1$. If exactly $n$ are $\leq 1$, the sequence is split exact, hence $a=0$ and all $a_{i}$ 's are $\leq 1$, contradicting the assumption. Hence at least $n+1$ of the $a_{i}$ 's are $\leq 1$. If they are all $\leq 1$, then clearly $a \leq 1$ as well, and in this case, we have

$$
\mathcal{G}_{\ell} \cong n \mathcal{O}_{\ell}(-1) \oplus 2 \mathcal{O}_{\ell}
$$


If say $a_{1} \geq 2$ and of the remaining, $p$ of the $a_{i}$ 's are 1's with the rest zeroes, then the cokernel of $\alpha_{\ell}$ is $\mathcal{O}_{\ell}\left(-a_{1}\right) \oplus \mathcal{O}_{\ell}(n-p)$, hence in this case $a=a_{1}$ and $p=n-a$. So in this case (when $a \geq 2$ ) we get

$$
\mathcal{G}_{\ell} \cong \mathcal{O}_{\ell}(-a) \oplus(n-a) \mathcal{O}_{\ell}(-1) \oplus(a+1) \mathcal{O}_{\ell}
$$

The largest possible value for $a$ is $n$, and in this case we say that $\ell$ is a jumping line of maximal order for $\mathcal{E}$. In any event, if $\ell$ with equations $\left\{X_{0}=X_{1}=0\right\}$ is a jumping line for $\mathcal{E}$ of order $a \geq 2$, from the decomposition of $\mathcal{G}_{\ell}$, we see that after a change of basis, $(2 n+2) \mathcal{O}_{\mathbf{P}^{3}}$ can be decomposed as $(a+1) \mathcal{O}_{\mathbf{P}^{3}} \oplus(2 n+1-a) \mathcal{O}_{\mathbf{P}^{3}}$, with $\beta=\left(\phi_{1}, \phi_{2}\right)$, where $\phi_{1}$ is a matrix over $R=k\left[X_{0}, X_{1}\right]$.

\section{$\S 2$.}

Suppose that $\mathcal{E}$ has a jumping line $\ell$ (with equations $X_{0}=X_{1}=0$, say) of order $n$. From the splitting of $\mathcal{G}$, this happens if and only if we can decompose $(2 n+2) \mathcal{O}_{\mathbf{P}^{3}}$ as $(n+1) \mathcal{O}_{\mathbf{P}^{3}} \oplus(n+1) \mathcal{O}_{\mathbf{P}^{3}}$ in such a way that $\beta=\left(\phi_{1}, \phi_{2}\right)$, where $\phi_{1}$ is a matrix over $R=k\left[X_{0}, X_{1}\right]$. Let $P$ be the mapping $(n+1) \mathcal{O}_{\mathbf{P}^{1}}(-1) \rightarrow n \mathcal{O}_{\mathbf{P}^{1}}$ given by $\phi_{1}$.

Claim. The homomorphism on $\mathbf{P}^{1},(n+1) \mathcal{O}_{\mathbf{P}^{1}}(-1) \stackrel{P}{\rightarrow} n \mathcal{O}_{\mathbf{P}^{1}}$ is onto.

Proof. Let $D$ be the cokernel of $P . H^{0}\left(n \mathcal{O}_{\mathbf{P}^{1}}\right)$ surjects onto $H^{0}(D)$. Suppose that $D$ is not zero. Then $D$ is a direct sum of line bundles on $\mathbf{P}^{1}$ of non-negative degrees and of modules with finite support and is regular in the sense of Castelnuovo-Mumford. We thus get the diagram (where $b=h^{0}(D)$ is not zero)

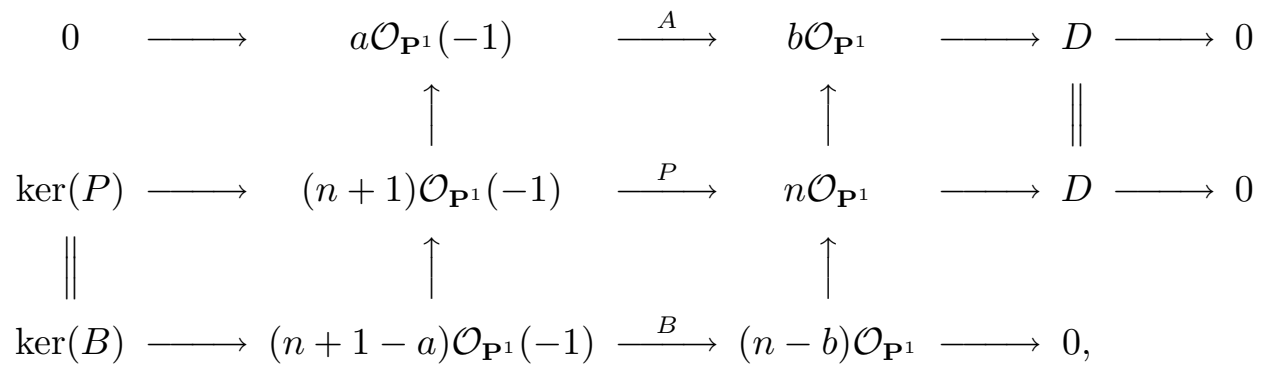

with split exact vertical sequences, and with $a \leq b$. Therefore after a change of basis, $P=\left[\begin{array}{ll}A & 0 \\ C & B\end{array}\right]$. First of all $b<n$, for otherwise, if $b=n$, since $n+1-a>0$, then $P$ has a column of zeroes. Going back to the matrix $\phi_{1}$ and to $\beta$ on $\mathbf{P}^{3}$, we conclude that $\beta$ has a column of zeroes which contradicts the minimality of the monad for $\mathcal{E}$. 
Now $B$ is onto, so we may decompose it as $\left[B_{1}, B_{2}\right]$ where $B_{1}$ is $(n-b) \times$ $(n-b+1)$ and is itself onto. The kernel of $B_{1}$ is $\mathcal{O}_{\mathbf{P}^{1}}(n-b+1)$, the dual map $(n-b+1) \mathcal{O}_{\mathbf{P}^{1}} \rightarrow \mathcal{O}_{\mathbf{P}^{1}}(n-b)$ is onto on the level of global sections, hence $B_{1}^{\vee}$ is just a presentation of the forms on $\mathbf{P}^{1}$ of degree $n-b$. Hence for suitable bases, we may write $B_{1}$ as

$$
B_{1}=\left[\begin{array}{ccccc}
X_{0} & X_{1} & 0 & \ldots & 0 \\
0 & X_{0} & X_{1} & \ldots & 0 \\
. & . & . & \ldots & . \\
. & . & . & \ldots & . \\
0 & 0 & 0 & \ldots & X_{1}
\end{array}\right]
$$

Clearly, by column operations, we can conclude that $B_{2}$ has only multiples of $X_{0}$ appearing in it. We now claim that $a=b$. For if $a<b$, then $B_{2}$ has at least one column, which cannot be zero by the earlier argument that $\beta$ cannot have a zero column. So $B_{2}$ has a column involving only $X_{0}$ which is independent from the first column of $B_{1}$. Conveying these two columns back to columns of $\beta$, we see that in the presentation of $H_{*}^{1}(\mathcal{E})$ given by $\beta$, there are 2 linearly independent elements of $H^{1}(\mathcal{E}(-1))$ which are annihilated by $X_{0}$. Looking at the restriction sequence to the plane $H=\left\{X_{0}=0\right\}$,

$$
0 \rightarrow \mathcal{E}(-1) \stackrel{X_{0}}{\longrightarrow} \mathcal{E} \rightarrow \mathcal{E}_{H} \rightarrow 0,
$$

this tells us that $\mathcal{E}_{H}$ has two independent sections in degree zero and none in lower degrees. This is not possible for a non-split bundle of first Chern class 0 like $\mathcal{E}_{H}$.

So we must have $b<n, a=b$. Hence $D$ has finite support. Without loss of generality, let us assume that one of the points in the support of $D$ is $X_{0}=0$. By the structure theorem for modules over a principal ideal domain, there is an element of $D$ which is annihilated by $X_{0}$. Hence after a change of basis, we may assume that $A$ has a column with only $X_{0}$ appearing in it, and by column operations using $B$, we may assume this entire column of $P$ has only $X_{0}$ appearing in it. This leads to the same contradiction as before. Thus $D$ must be zero.

Theorem. If $\mathcal{E}$ is an instanton bundle on $\mathbf{P}^{3}$ with a jumping line of maximal order, then $H^{2}(\mathcal{E} \otimes \mathcal{E}(-1))=0$.

Proof. If E has a jumping line of maximal order $n$, where $n=c_{1}(\mathcal{E})$, by the above discussion, the monad map $\beta$ can be decomposed as $\phi_{1}, \phi_{2}$, where $\phi_{1}$ is a matrix over $R=k\left[X_{0}, X_{1}\right]$, say, or is a map on $\mathbf{P}^{1}$ giving a surjection $(n+1) \mathcal{O}_{\mathbf{P}^{1}}(-1) \stackrel{\phi_{1}}{\longrightarrow} n \mathcal{O}_{\mathbf{P}^{1}}$. Consider the dual sequence

$$
0 \rightarrow n \mathcal{O}_{\mathbf{P}^{1}}(-1) \stackrel{\phi_{1}^{\vee}}{\longrightarrow}(n+1) \mathcal{O}_{\mathbf{P}^{1}} \rightarrow \mathcal{O}_{\mathbf{P}^{1}}(n) \rightarrow 0 .
$$


We get the exact Eagon-Northcott sequence

$$
\begin{aligned}
0 \rightarrow \wedge^{2}\left(n \mathcal{O}_{\mathbf{P}^{1}}(-1)\right) \stackrel{F_{1}^{\vee}}{\rightarrow} n \mathcal{O}_{\mathbf{P}^{1}}(-1) & \otimes(n+1) \mathcal{O}_{\mathbf{P}^{1}} \\
& \rightarrow S_{2}\left((n+1) \mathcal{O}_{\mathbf{P}^{1}}\right) \rightarrow \mathcal{O}_{\mathbf{P}^{1}}(2 n) \rightarrow 0 .
\end{aligned}
$$

Dually we get

$$
\begin{aligned}
0 \rightarrow \mathcal{O}_{\mathbf{P}^{1}}(-2 n) \rightarrow S_{2}(( & \left.n+1) \mathcal{O}_{\mathbf{P}^{1}}\right) \\
& \rightarrow(n+1) \mathcal{O}_{\mathbf{P}^{1}} \otimes n \mathcal{O}_{\mathbf{P}^{1}}(1) \stackrel{F_{1}}{\longrightarrow} \wedge^{2}\left(n \mathcal{O}_{\mathbf{P}^{1}}(1)\right) \rightarrow 0
\end{aligned}
$$

The kernel of $F_{1}$ is a bundle $\mathcal{B}_{1}$ on $\mathbf{P}^{1}$ which is clearly a direct sum of $\mathcal{O}_{\mathbf{P}^{1}}$ 's and $\mathcal{O}_{\mathbf{P}^{1}}(1)$ 's. Hence the module $N_{R}$ is of finite length with nonzero gradings only in degree -2 . It follows that for the module $M$ as well, $M_{-1}=0$.

$\S 3$.

Suppose that $\mathcal{E}$ has a jumping line $\ell=\left\{X_{0}=X_{1}=0\right\}$ of order $n-1$. Then $\beta$ can be decomposed as $\left(\phi_{1}, \phi_{2}\right)$ where $\phi_{1}$ is an $n \times n$ matrix with entries in $R=k\left[X_{0}, X_{1}\right]$. As in the prevous situation, we let $P$ denote the homomorphism on $\mathbf{P}^{1}$ given by $\phi_{1}$, and let $D$ denote the cokernel of $n \mathcal{O}_{\mathbf{P}^{1}}(-1) \stackrel{P}{\rightarrow} n \mathcal{O}_{\mathbf{P}^{1}}$, (though now $D \neq 0$ ) and get a diagram

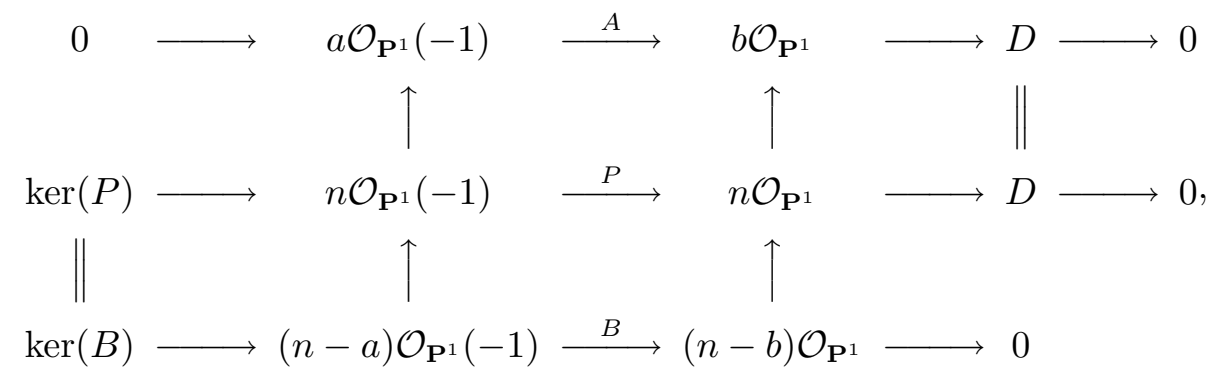

with split exact vertical sequences, with $b \neq 0$ and with $a \leq b$. Therefore after a change of basis, $P=\left[\begin{array}{ll}A & 0 \\ C & B\end{array}\right]$.

First let's make the following easy observation: If $\sigma: V \rightarrow W$ is a vector space mapping with kernel of dimension one, then the induced map $S$ : $\wedge^{2} V \rightarrow V \otimes W$ is an injection. $\left(S\left(\sum a_{i} \wedge b_{i}\right)=\sum\left(a_{i} \otimes \sigma\left(b_{i}\right)-b_{i} \otimes \sigma\left(a_{i}\right)\right)\right.$.)

Case 1. $b=n$.

If so, since $P$ cannot have a column of zeros, we get $a=n$. Then $P$ : $n \mathcal{O}_{\mathbf{P}^{1}}(-1) \rightarrow n \mathcal{O}_{\mathbf{P}^{1}}$ is an inclusion of sheaves, with cokernel $D$ of finite support on $\mathbf{P}^{1}$. By the structure theorem for modules over a principal ideal 
domain, $D$ is a direct sum of cyclic modules supported at various points of $\mathbf{P}^{1}$. We claim that each point of $\mathbf{P}^{1}$ supports at most one such cyclic module. For if two summands of $D$ are supported at a point $x$ given by say $X_{0}=0$, then there are two distinct elements of $D$, one obtained from each summand, which are annihilated by $X_{0}$. As in the previous arguments, this gives (after a change of basis), two independent columns of $P$ containing only $X_{0}$ 's, a contradiction. Hence we may conclude that $P$ is of corank 0 or 1 at any point $x$.

Consider the dual map $n \mathcal{O}_{\mathbf{P}^{1}}(-1) \stackrel{P^{\vee}}{\longrightarrow} n \mathcal{O}_{\mathbf{P}^{1}}$ and the associated EagonNorthcott complex

$$
0 \rightarrow \wedge^{2}\left(n \mathcal{O}_{\mathbf{P}^{1}}(-1)\right) \stackrel{Q_{1}}{\longrightarrow} n \mathcal{O}_{\mathbf{P}^{1}}(-1) \otimes n \mathcal{O}_{\mathbf{P}^{1}} \stackrel{Q_{2}}{\longrightarrow} S_{2}\left(n \mathcal{O}_{\mathbf{P}^{1}}\right)
$$

This complex is exact outside of the support of $D$, and by the observations made above, $Q_{1}$ is a bundle injection. Hence this complex is exact at all points of $\mathbf{P}^{1}$. Let $\mathcal{B}_{1}^{\vee}$ be the image of $Q_{2}$. It is a direct sum of $\mathcal{O}_{\mathbf{P}^{1}}$ 's and $\mathcal{O}_{\mathbf{P}^{1}}(-1)^{\prime}$ 's. Dually, we get the exact sequence

$$
0 \rightarrow \mathcal{B}_{1} \rightarrow n \mathcal{O}_{\mathbf{P}^{1}} \otimes n \mathcal{O}_{\mathbf{P}^{1}}(1) \stackrel{F_{1}}{\longrightarrow} \wedge^{2}\left(n \mathcal{O}_{\mathbf{P}^{1}}(1)\right) \rightarrow 0
$$

Hence $N_{R}$ (the cokernel of $F_{1}$ ) is zero in degrees -1 and 0 . It follows that $H^{2}(\mathcal{E} \otimes \mathcal{E}(-1))$ and $H^{2}(\mathcal{E} \otimes \mathcal{E})$ are both zero.

Case 2. $0<b<n$.

Then $n-a \geq n-b+1$ and in fact if it is strictly greater, then $B=\left(B_{1}, B_{2}\right)$ and this leads to a contradiction as in the earlier argument with a jumping line of order $n$. So $n-a=n-b+1$, and $a=b-1$. If $D$ has a summand of finite support, as usual we argue that $A$ gets a column with only, say $X_{0}$ in it and thus together with a column from $B$, we see that $P$ has two columns with only $X_{0}$ 's appearing, leading to a contradiction. So $D$ is the line bundle $\mathcal{O}_{\mathbf{P}^{1}}(b-1)$, and the kernel and cokernel of $P$ can be identified as

$$
0 \rightarrow \mathcal{O}_{\mathbf{P}^{1}}(b-n-1) \rightarrow n \mathcal{O}_{\mathbf{P}^{1}}(-1) \stackrel{P}{\rightarrow} n \mathcal{O}_{\mathbf{P}^{1}} \rightarrow \mathcal{O}_{\mathbf{P}^{1}}(b-1) \rightarrow 0
$$

Then $P$ has corank 1 at each point of $\mathbf{P}^{1}$. The dual sequence gives

$$
0 \rightarrow \mathcal{O}_{\mathbf{P}^{1}}(-b) \rightarrow n \mathcal{O}_{\mathbf{P}^{1}}(-1) \stackrel{P^{\vee}}{\rightarrow} n \mathcal{O}_{\mathbf{P}^{1}} \rightarrow \mathcal{O}_{\mathbf{P}^{1}}(n-b) \rightarrow 0,
$$

and let $\mathcal{A}$ be the image of $P^{\vee}$. Then the top and bottom rows of the following 
commuting diagram are exact:

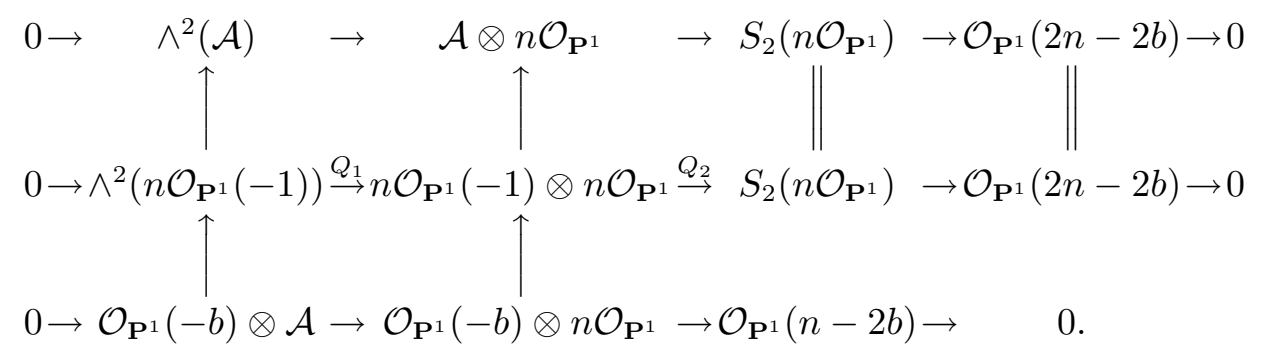

The second row is a complex with $Q_{1}$ a bundle injection, and with exactness at $S_{2}\left(n \mathcal{O}_{\mathbf{P}^{1}}\right)$. As usual, let $N_{R}$ denote the $R$-module equal to the cokernel of $H_{*}^{0}\left(Q_{1}^{\vee}\right)$. A diagram chase shows that in degrees -1 and 0 , the graded pieces of $N_{R}$ have dimension equal to $h^{1}\left(\mathcal{O}_{\mathbf{P}^{1}}(2 b-n-1)\right)$ and $h^{1}\left(\mathcal{O}_{\mathbf{P}^{1}}(2 b-n)\right)$ respectively. Thus for such a bundle $\mathcal{E}$,

$$
h^{2}(\mathcal{E} \otimes \mathcal{E}(-1)) \leq \max \{n-2 b, 0\}, h^{2}(\mathcal{E} \otimes \mathcal{E}) \leq \max \{n-2 b-1,0\}
$$

Theorem. A mathematical instanton bundle with $c_{2}=5$ and with a jumping line of order 4 is unobstructed.

Proof. According to Le Potier ([L, Proposition 11]), if $h^{2}(\mathcal{E} \otimes \mathcal{E}) \neq 0$, then $h^{2}(\mathcal{E} \otimes \mathcal{E}(-1)) \geq 4$. According to the above discussion, if $b=5$, then $\mathcal{E}$ is unobstructed. If $h^{2}(\mathcal{E} \otimes \mathcal{E}) \neq 0$ then we must have $5-2 b \geq 4$. This happens only if $b=0$ which is not possible.

\section{$\S 4$.}

We will give a description of how bundles with maximal order jumping lines arise. (However, we do not give a construction for bundles with jumping lines of order one less than the maximal order.)

Proposition. Let $C$ be the $(n-1)$-th infinitesimal neighborhood of the line $\ell: X_{0}=X_{1}=0$, defined by the ideal $I(C)=\left(X_{0}, X_{1}\right)^{n}$. The set of isomorphism classes of mathematical instanton bundles with $c_{1}=0, c_{2}=n$ and with $\ell$ a jumping line of order $n$ are in one-to one correspondence with the set of surjective homomorphisms $t: \mathcal{I}_{C}(n) \rightarrow \omega_{C}(4-n)$ modulo the 
equivalence relation that identifies $t$ with any nonzero scalar multiple of $t$.

Proof. First of all we will construct one such bundle for any given line $\ell$. Let $\phi, \psi$ be the $n \times(n+1)$ matrices

$$
\phi=\left[\begin{array}{ccccc}
X_{0} & X_{1} & 0 & \ldots & 0 \\
0 & X_{0} & X_{1} & \ldots & 0 \\
. & . & . & \ldots & . \\
. & . & . & \ldots & . \\
0 & 0 & 0 & \ldots & X_{1}
\end{array}\right], \quad \psi=\left[\begin{array}{ccccc}
0 & \ldots & 0 & X_{2} & X_{3} \\
0 & \ldots & X_{2} & X_{3} & 0 \\
. & \ldots & . & . & . \\
. & \ldots & . & . & . \\
X_{2} & \ldots & 0 & 0 & 0
\end{array}\right]
$$

Then the following is clearly a monad, giving rise to an instanton bundle with $c_{1}=0, c_{2}=n$ and with $\ell$ as a jumping line of order $n$ (and also another jumping line $X_{2}=X_{3}=0$ of order $n$ ):

$$
0 \rightarrow n \mathcal{O}_{\mathbf{P}^{3}}(-1) \stackrel{\left[\begin{array}{c}
-\psi^{\vee} \\
\phi^{\vee}
\end{array}\right]}{\longrightarrow}(n+1) \mathcal{O}_{\mathbf{P}^{3}} \oplus(n+1) \mathcal{O}_{\mathbf{P}^{3}} \stackrel{[\phi \psi]}{\longrightarrow} n \mathcal{O}_{\mathbf{P}^{3}}(1) \rightarrow 0 .
$$

Hence such bundles do exist. (See Remark 1.)

Now let $\mathcal{E}$ be any instanton bundle with $c_{1}=0, c_{2}=n$ and with $\ell$ as a jumping line of order $n$. It has a monad which can be decomposed as

$$
\left.0 \rightarrow n \mathcal{O}_{\mathbf{P}^{3}}(-1) \stackrel{\left[\phi^{\prime}\right.}{\psi^{\prime}}\right] \text { }(n+1) \mathcal{O}_{\mathbf{P}^{3}} \oplus(n+1) \mathcal{O}_{\mathbf{P}^{3}} \stackrel{[\phi \psi]}{\longrightarrow} n \mathcal{O}_{\mathbf{P}^{3}}(1) \rightarrow 0,
$$

where $\phi=\left[\begin{array}{ccccc}X_{0} & X_{1} & 0 & \ldots & 0 \\ 0 & X_{0} & X_{1} & \ldots & 0 \\ \cdot & \cdot & . & \ldots & . \\ . & . & . & \ldots & . \\ 0 & 0 & 0 & \ldots & X_{1}\end{array}\right]$. This follows from the Claim in $\S 2$. We may write down a commuting square

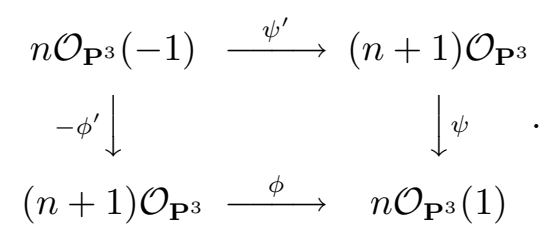

The map $\phi$ defines the curve $C$ by means of its maximal minors. We claim that so does $\psi^{\prime}$. To see this, since $\phi \equiv 0$ modulo $\left(X_{0}, X_{1}\right), \psi$ has rank $n$ at 
each point of $\ell$. Let $\bar{\psi}=\psi$ modulo $\left(X_{0}, X_{1}\right)$. Then as a matrix in $X_{2}$ and $X_{3}$, $\bar{\psi}$ fits into the exact sequence

$$
0 \rightarrow \mathcal{O}_{\mathbf{P}^{1}}(-n) \rightarrow(n+1) \mathcal{O}_{\mathbf{P}^{1}} \stackrel{\bar{\psi}}{\rightarrow} n \mathcal{O}_{\mathbf{P}^{1}}(1) \rightarrow 0 .
$$

From the equation $-\phi \phi^{\prime}=\psi \psi^{\prime}$, we get $\psi \psi^{\prime} \equiv 0$ modulo $\left(X_{0}, X_{1}\right)$. However the kernel of $\bar{\psi}$ has sections only in degree $n$, hence $\psi^{\prime} \equiv 0$ modulo $\left(X_{0}, X_{1}\right)$. Now the analysis of $\S 2$ applied to $\psi^{\prime \vee}$ in the dual monad shows that $\psi^{\prime}$ also defines the curve $C$ by means of its maximal minors. Hence the commutative diagram above can be completed to

$(*)$

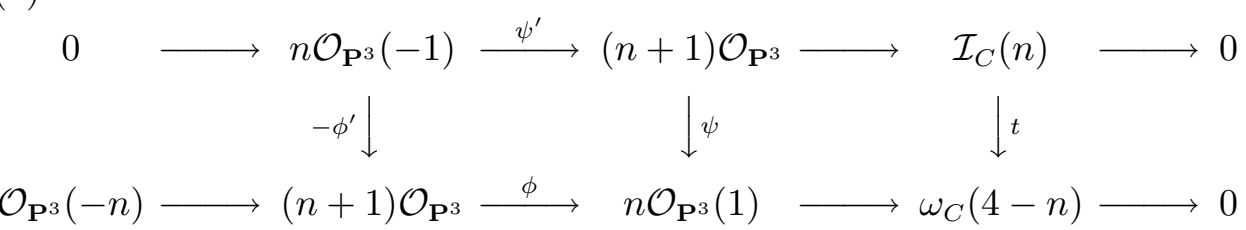

and the homomorphism $\mathcal{I}_{C}(n) \stackrel{t}{\rightarrow} \omega_{C}(4-n)$ is onto.

Now suppose $\tilde{\mathcal{E}}$ is another mathematical instanton bundle with $c_{1}=$ $0, c_{2}=n$ and with the same $\ell$ as jumping line of order $n$. Its monad can be split and we can assume that we get $\tilde{\phi}, \tilde{\psi}, \tilde{\phi}^{\prime}, \tilde{\psi}^{\prime}$ in the same way as $\phi, \psi, \phi^{\prime}, \psi^{\prime}$, and in fact we may assume after change of bases that $\tilde{\phi}=\phi$ and $\tilde{\psi}^{\prime}=\psi^{\prime}$. An isomorphism of $\mathcal{E}$ with $\tilde{\mathcal{E}}$ can be lifted to an isomorphism of monads, with

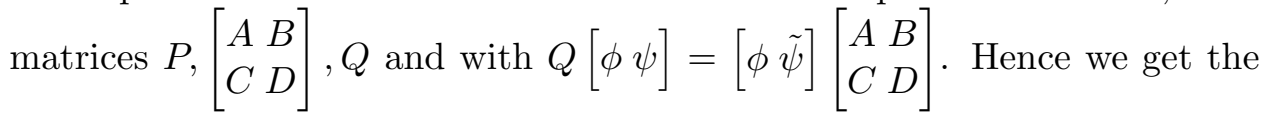
equation $Q \phi=\phi A+\tilde{\psi} C$. Modulo $\left(X_{0}, X_{1}\right)$, this gives $\tilde{\psi} C \equiv 0$, and since the kernel of $\tilde{\psi} \bmod \left(X_{0}, X_{1}\right)$ has sections only in degree $n$, we get $C=0$. So $Q \phi=\phi A$. Viewed on $\mathbf{P}^{1}, Q^{\vee}, A^{\vee}$ are the unique lifts of an automorphism of $\mathcal{O}_{\mathbf{P}^{1}}(n)$, hence they are scalar matrices $\lambda$. Likewise, $P, D$ are scalar matrices $\mu$. It follows that the nowhere vanishing sections $t, \tilde{t}$ given by the diagrams $(*)$ and its analogue for $\tilde{\mathcal{E}}$ satisfy $\lambda t=\mu \tilde{t}$, since $B$ provides a homotopy of the corresponding diagrams.

Thus if $\mathcal{E} \cong \tilde{\mathcal{E}}$ then the corresponding homomorphisms $t, \tilde{t}: \mathcal{I}_{C}(n) \rightarrow$ $\omega_{C}(4-n)$ differ by a nonzero scalar. Conversely, if $\mathcal{E}$ and $\tilde{\mathcal{E}}$ give rise to $t, \tilde{t}$ such that $t=\lambda \tilde{t}, \lambda \neq 0$, we see from $(*)$ that the lifts of $t$ and $\lambda \tilde{t}$ which are $\left(-\phi^{\prime}, \psi\right)$ and $\left(-\lambda \tilde{\phi}^{\prime}, \lambda \tilde{\psi}\right)$ must differ by a homotopy $B$, and we can recreate the isomorphism of monads and hence an isomorphism from $\mathcal{E}$ to $\tilde{\mathcal{E}}$.

We have seen that there is one $t: \mathcal{I}_{C}(n) \rightarrow \omega_{C}(4-n)$ which is surjective, since we showed the existence of one bundle with jumping line $\ell$ of order $n$. Let $V$ be the vector subspace of $\operatorname{Hom}\left((n+1) \mathcal{O}_{\mathbf{P}^{3}}, n \mathcal{O}_{\mathbf{P}^{3}}(1)\right)$ consisting of all homomorphisms which lift elements of $\operatorname{Hom}\left(\mathcal{I}_{C}(n), \omega_{C}(4-n)\right)$. Since every element of $\operatorname{Hom}\left(\mathcal{I}_{C}(n), \omega_{C}(4-n)\right)$ can be lifted, there is an open set in $V$ 
consisting of elements $\psi$ which lift surjective elements $t$ of $\operatorname{Hom}\left(\mathcal{I}_{C}(n), \omega_{C}(4-\right.$ $n))$. $\psi$ in turn gives rise to a unique $-\phi^{\prime}$ to get the commuting diagram

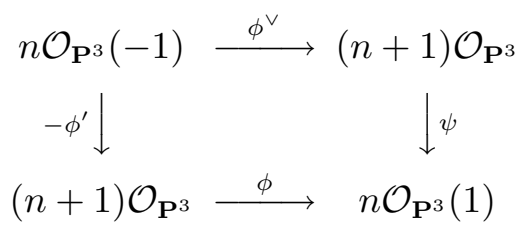

(the existence and uniqueness of $-\phi^{\prime}$ arising from the fact that the kernel of $\phi$ is $\left.\mathcal{O}_{\mathbf{P}^{3}}(-n)\right)$. Since there is one $\psi$ for which the pair of maps $(\phi, \psi),\left(\begin{array}{c}\phi^{\prime} \\ \phi^{\vee}\end{array}\right)$ is a monad, it follows that for the general choice of $\psi \in V$, the pair of maps $(\phi, \psi),\left(\begin{array}{c}\phi^{\prime} \\ \phi^{\vee}\end{array}\right)$ will also give a monad; that is to say, the map $\left(\begin{array}{c}\phi^{\prime} \\ \phi^{\vee}\end{array}\right)$ will also be an injection of vector bundles. Thus the general element of $\operatorname{Hom}\left(\mathcal{I}_{C}(n), \omega_{C}(4-n)\right)$ gives rise to a rank two bundle which is the homology of the monad thus constructed, and it is evident from the form of the monad that this bundle is a mathematical instanton bundle with $c_{1}=0, c_{2}=n$ and with $\ell$ as a jumping line of order $n$.

Remark 1. To the best of our knowledge, Peskine first looked at examples of type $(\dagger)$. In the monad written down, one can change degrees of the matrix entries. So one can replace the pair $X_{0}, X_{1}$ by any forms of the same degree and the pair $X_{2}, X_{3}$ by forms of the same degree so chosen that the four forms have no common zeros in $\mathbf{P}^{3}$, to still have a monad. In particular, keeping $X_{2}, X_{3}$ and replacing $X_{0}, X_{1}$ by forms of degree $2 n-1$, we get a monad $0 \rightarrow n \mathcal{O}_{\mathbf{P}^{3}}(-n) \rightarrow(n+1) \mathcal{O}_{\mathbf{P}^{3}}(-n+1) \oplus(n+1) \mathcal{O}_{\mathbf{P}^{3}}(n-1) \rightarrow n \mathcal{O}_{\mathbf{P}^{3}}(n) \rightarrow 0$. This gives a stable bundle with $X_{2}=X_{3}=0$ as a jumping line of order $2 n^{2}-1$, with $c_{2}=n^{2}+n-1$, hence an extremal example for which the order of the jumping line is equal to $2 c_{2}+2-\sqrt{4 c_{2}+5}$, the bound obtained by Chang $[\mathbf{C}]$.

Remark 2. The construction described above should be called a Ferrand construction since it is similar to the construction of Ferrand $[\mathbf{F}]$ on locally complete intersection curves. In fact, looking at $(*)$, we see that the map from $\mathcal{O}_{\mathbf{P}^{3}}(-n)$ results in a section of $\mathcal{E}(n)$, and the zero scheme of this section is just the curve whose ideal sheaf is given by the kernel of $t$. So the construction gives a subcanonical double structure on $C$, where unlike in Ferrand's situation, $C$ is not a locally complete intersection curve.

We can now estimate the size of the set of mathematical instanton bundles with jumping line of order $n$. We will compute the dimension of the vector 
space $\operatorname{Hom}\left(\mathcal{I}_{C}(n), \omega_{C}(4-n)\right)$ and it will then suffice to see whether the same $\mathcal{E}$ can be obtained through a different jumping line of order $n$. We will see below that this cannot happen in general for $n \geq 3$, hence the dimension of the set of instanton bundles with jumping lines of order $n$ equals the dimension of the set of lines in $\mathbf{P}^{3}+$ the dimension of $\operatorname{Hom}\left(\mathcal{I}_{C}(n), \omega_{C}(4-n)\right)$ -1 .

Proposition. If $C$ is the curve with $I(C)=\left(X_{0}, X_{1}\right)^{n}, n>1$, then the dimension of $\operatorname{Hom}\left(\mathcal{I}_{C}(n), \omega_{C}(4-n)\right)$ is $6 n-1$, and the general homomorphism in this space gives rise to an instanton bundle with a unique jumping line of order $n$ (if $n \geq 3$ ). Hence the set of instanton bundles with $c_{1}=0, c_{2}=n \geq 3$ and with a jumping line of order $n$ has dimension equal to $6 n+2$.

Proof. (i) Let $r$ be an integer with $0 \leq 1-r<n$. Since there is no nonzero map from $n \mathcal{O}_{\mathbf{P}^{3}}(-1)$ to $\operatorname{ker}(\phi)$, an element of $\operatorname{Hom}\left(\mathcal{I}_{C}(n), \omega_{C}(4-n-r)\right)$ is equivalent to a homotopy equivalence class of morphisms of complexes

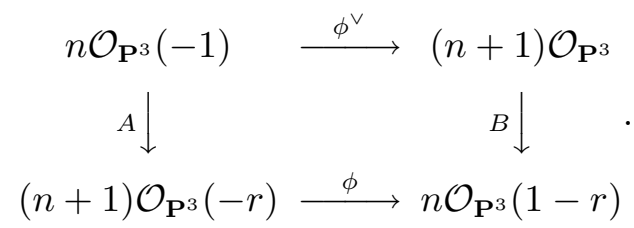

We start by considering the problem over $\mathbf{P}^{1}$ (insisting that $A$ and $B$ have entries only involving $\left.X_{0}, X_{1}\right)$. Viewed as such, the diagram induces a connecting morphism $H^{0}\left(\operatorname{cok}\left(\phi^{\vee}\right)\right) \rightarrow H^{1}(\operatorname{ker}(\phi))$, or after twisting down appropriately, $H^{0}\left(\mathcal{O}_{\mathbf{P}^{1}}\right) \rightarrow H^{1}\left(\mathcal{O}_{\mathbf{P}^{1}}(-2 n-r)\right)$. It is straightforward to verify that homotopy classes of these complexes over $\mathbf{P}^{1}$ are in one-to-one correspondence with elements of $H^{1}\left(\mathcal{O}_{\mathbf{P}^{1}}(-2 n-r)\right)$. Now morphisms over $\mathbf{P}^{3}$ can be obtained by combinations of morphisms over $\mathbf{P}^{1}$ using the other variables $X_{2}, X_{3}$. Hence

$\operatorname{dim} \operatorname{Hom}\left(\mathcal{I}_{C}(n), \omega_{C}(3-n)\right)=h^{1}\left(\mathcal{O}_{\mathbf{P}^{1}}(-2 n-1)\right)=2 n$ and

$\operatorname{dim} \operatorname{Hom}\left(\mathcal{I}_{C}(n), \omega_{C}(4-n)\right)=h^{1}\left(\mathcal{O}_{\mathbf{P}^{1}}(-2 n)\right)+2 h^{1}\left(\mathcal{O}_{\mathbf{P}^{1}}(-2 n-1)\right)=2 n-$ $1+2 n+2 n=6 n-1$.

(ii) Suppose a bundle $\mathcal{E}$ has two jumping lines $\ell, \ell^{\prime}$ of order $n$. Suppose that $\ell$ and $\ell^{\prime}$ are not disjoint, with the point $x$ in common. Then if we think of the map $\beta$ in the monad for $\mathcal{E}$ as a map $V \otimes_{k} \mathcal{O}_{\mathbf{P}^{3}} \stackrel{\beta}{\rightarrow} n \mathcal{O}_{\mathbf{P}^{3}}(1)$, we get two subspaces $W, W^{\prime}$ of $V$, both of dimension $n+1$, such that $\beta$ restricted to $W \otimes_{k} \mathcal{O}_{\mathbf{P}^{3}}$ is a matrix with entries in the ideal of $\ell$, and likewise for $W^{\prime}$. Hence $\beta$ restricted to $W+W^{\prime}$ vanishes at $x$. Therefore the dimension of $W+W^{\prime}$ must be $\leq n+2$, since $\beta$ is surjective at $x . \beta$ restricted to $W \cap W^{\prime}$ has entries involving only the form $H$ of the plane spanned by $\ell, \ell^{\prime}$. Hence, viewed as a map on $\mathbf{P}^{1}, \beta$ restricted to $W$ has kernel equal to $W \cap W^{\prime}$ at the 
point $H=0$ of $\mathbf{P}^{1}$. But $\beta$ restricted to $W$ is the map $\phi_{1}$ studied in $\S 2$, which was onto when viewed as a map on $\mathbf{P}^{1}$. Hence $W \cap W^{\prime}$ is one dimensional, which is a contradiction. Therefore $\ell$ and $\ell^{\prime}$ must be disjoint.

Let $\ell$ have equations $X_{0}=X_{1}=0$ and $\ell^{\prime}$ have equations $X_{2}=X_{3}=0$. There is a decomposition of $\beta$ as $\left(\phi_{1}, \phi_{2}\right)$ where $\phi_{1}$ has only $X_{0}, X_{1}$ and $\phi_{2}$ has only $X_{2}, X_{3}$. The induced element of $\operatorname{Hom}\left(\mathcal{I}_{C}(n), \omega_{C}(4-n)\right)$ then has the form $X_{2} t+X_{3} t^{\prime}$. Hence it suffices to show that the general element of $\operatorname{Hom}\left(\mathcal{I}_{C}(n), \omega_{C}(4-n)\right)$ cannot be written in the form $X_{2}^{\prime} u+X_{3}^{\prime} u^{\prime}$, for any choice of $u, u^{\prime}$ and $X_{2}^{\prime}, X_{3}^{\prime}$ (complementing $X_{0}, X_{1}$ ). (In fact the element can be a general perturbation of the section $X_{2} t+X_{3} t^{\prime}$, and since this will be a surjective homomorphism, it gives rise to a corresponding bundle with only $\ell$ as a jumping line of order $n$.)

Let $t, t^{\prime}$ be linearly independent in $\operatorname{Hom}\left(\mathcal{I}_{C}(n), \omega_{C}(3-n)\right)$ and choose $r \in \operatorname{Hom}\left(\mathcal{I}_{C}(n), \omega_{C}(4-n)\right)$ of the form $X_{0} r_{0}+X_{1} r_{1}$ but which is not in the span of multiples of $t, t^{\prime}$ by $X_{0}, X_{1}$ (since $n \geq 3$, this can be done). Then we claim that $r+X_{2} t+X_{3} t^{\prime}$ cannot be written as $X_{2}^{\prime} u+X_{3}^{\prime} u^{\prime}$. For suppose they are equal. Since $X_{2}^{\prime}=a\left(X_{0}, X_{1}\right)+b\left(X_{2}, X_{3}\right), X_{3}^{\prime}=c\left(X_{0}, X_{1}\right)+d\left(X_{2}, X_{3}\right)$, we see that

$$
\begin{aligned}
X_{2} t+X_{3} t^{\prime} & =b\left(X_{2}, X_{3}\right) u+d\left(X_{2}, X_{3}\right) u^{\prime} \\
X_{0} r_{0}+X_{1} r_{1} & =a\left(X_{0}, X_{1}\right) u+c\left(X_{0}, X_{1}\right) u^{\prime} .
\end{aligned}
$$

Since there are no nontrivial relationships involving only $X_{2}, X_{3}$ we see that $t, t^{\prime}$ are scalar combinations of $u, u^{\prime}$, and since $t, t^{\prime}$ span a two dimensional space, conversely $u, u^{\prime}$ are scalar combinations of $t, t^{\prime}$. Hence $r$ is in the span of multiples of $t, t^{\prime}$ by $X_{0}, X_{1}$, against our assumption.

\section{References}

[A-O] V. Ancona and G. Ottaviani, On singularities of $M_{\mathbf{P}^{3}}\left(c_{1}, c_{2}\right)$, preprint, 1995.

[C] M.C. Chang, A bound on the order of jumping lines, Math. Ann., 262 (1983), 511-516.

[D-V] A. Douady and J.-L. Verdier, Les équations de Yang-Mills, Astérisque, 71-72 (1980).

[F] D. Ferrand, Courbes gauche et fibres de rang 2, C.R. Acad. Sci. Paris, A281 (1975), 345-347.

[H] R. Hartshorne, Stable vector bundles of rank 2 on $\mathbf{P}^{3}$, Math. Ann., 238 (1978), 229-280.

[H-N] A. Hirschowitz and M.S. Narasimhan, Fibres de 't Hooft speciaux et applications, Progress in Math., 24, Birkhäuser (1981), 143-164.

[L] J. Le Potier, Sur l'espace de modules des fibrés de Yang et Mills, Progress in Math., 37, Birkhäuser (1983), 65-138. 
[N-T] T. Nüßler and G. Trautmann, Multiple Koszul structures on lines and instanton bundles, Int. J. Math., 5 (1994), 373-388.

[S] M. Skiti, Sur l'espace de modules des instantons mathématiques, C.R. Acad. Sci. Paris, 320(I) (1995), 1221-1224.

Received August 1, 1995 and revised June 7, 1996.

UNIVERSITY OF Missouri

ST. LOUIS, MO 63121-4499

E-mail address: rao@arch.umsl.edu 